\title{
Safety and efficacy data of a randomized, double-blind, placebo-controlled phase II study with the biological response modifier "bRESCAP" in patients undergoing cardiac surgery (APPIRED II)
}

\author{
Hiskias Keizer ${ }^{1}$, Suzanne Kats ${ }^{2}$, Ines Christis ${ }^{3}$, Rene Heylen ${ }^{3}$, Ronald Oosting ${ }^{1,4}$, Willem Seinen ${ }^{1,4}$ and Ruud Brands ${ }^{1,4}$ \\ ${ }^{1}$ Alloksys Life Sciences BV, Wageningen, The Netherlands \\ ${ }^{2}$ Department of Cardiothoracic Surgery, MUMC, Maastricht, The Netherlands \\ ${ }^{3}$ Department of Anesthesiology, Intensive Care and Emergency Medicine, ZOL Genk, Belgium \\ ${ }^{4}$ Institute for Risk Assessment, Utrecht University, Utrecht, Utrecht, The Netherlands
}

\begin{abstract}
The APPIRED II study is a randomized controlled phase II clinical study that was set up to test the anti-inflammatory effects of intervenously-dosed bRESCAP in 53 patients undergoing cardiac surgery. bRESCAP is a specific type of bovine intestinal alkaline phosphatase that is currently under development as a biological response modifier for the treatment of various inflammatory diseases.

Patients undergoing cardiac surgery are at increased risk to develop a systemic inflammatory response syndrome (SIRS). This syndrome may lead to death by downstream complications like multi-organ failure. In a previous study among patients undergoing coronary artery bypass grafting, it was demonstrated that bRESCAP diminishes the cytokine storm which appeared to be initiated by a significant TNFa response.

The current APPIRED II study was set up to further extend these findings in a population of cardiac surgery patients with a higher chance to die from surgery.

In APPIRED II, however no significant TNF alpha response occurred. Various other inflammatory markers in plasma of patients were temporarily increased upon surgery but did not respond to bRESCAP treatment.

Safety-wise, APPIRED II was successful. No statistically significant differences were observed between placebo treated and bRESCAP treated patients with respect to inflammatory cytokines, haematology, clinical chemistry, cardiac function, body temperature, hospitalization time and the total number of adverse effects. Perioperative mortality was absent in the bRESCAP group. In the placebo group, the perioperative mortality was $12 \%$ (n.s.).

From the data of the APPIRED II study it can be concluded that short term intravenous treatment of cardiac surgery patients with bRESCAP is safe. Potential TNF alpha lowering effects of bRESCAP treatment could not be demonstrated in this study as no TNF alpha dependent inflammatory responses were observed in any of the patients.
\end{abstract}

\begin{abstract}
Abbreviations: APPIRED: Alkaline Phosphatase in Prevention of Ischemia Reperfusion Damage, bIAP: bovine Alkaline Phosphatase, bRESCAP: bovine Rescuing Alkaline Phosphatase, CABG: Coronay Artery Bypass Grafting, SIRS: Systemic Inflammatory Response Syndrome
\end{abstract}

\section{Introduction}

During coronary artery cardiac bypass surgery, with the use of cardiopulmonary bypass, inflammatory responses often occur $[1,2]$.

There can be various reasons for this effect. First of all, general endothelial stress may occur in the body when the natural pulsatile aortic flow is changed into a laminar flow during the cardiac surgical procedure with the use of cardiopulmonary bypass [2]. Under these conditions surface receptors are stimulated on the endothelial cells which can activate the cells of the immune system. Secondly, postischemic oxidative reperfusion damage may occur in endothelia of the aorta and of myocytes. This leads to the release of cellular stress factors, like ATP, which in turn contributes to the formation of cytokines by the immune system [2]. Also, activation of white blood cells, by contacting the extracorporeal loop is a known trigger of the proinflammatory state [3].

Apart from these effects during cardiac surgery, transient ischemia of the gut occurs and may cause an increase in intestinal permeability. This induces an increase in systemic exposure to bacterial toxins like LPS [4,5] which has an activating effect on the immune system [2]. Altogether, these effects can culminate into the development of

${ }^{\star}$ Correspondence to: Hiskias Keizer, Alloksys Life Sciences BV, Wageningen, The Netherlands, E-mail: Hiskias@Alloksys.com

Key words: cytokines, inflammation, alkaline phosphatase, safety, efficacy, clinical trial

Received: May 05, 2021; Accepted: May 20, 2021; Published: May 30, 2021 
Keizer H (2021) Safety and efficacy data of a randomized, double-blind, placebo-controlled phase II study with the biological response modifier "bRESCAP" in patients undergoing cardiac surgery (APPIRED II)

a systemic inflammatory response syndrome (SIRS). This syndrome often starts with tachycardia and can cause renal failure, pulmonary failure, cardiac failure and neurological dysfunction. Such a multi organ failure often leads to death [2].

Alkaline Phosphatase (AP) is an enzyme which detoxifies bacterial toxins such as LPS [6] and inactivates cellular stress factors such as ATP [7]. Alkaline phosphatase is induced in vivo upon inflammation [6] and it is highly expressed on cells lining all physiological barriers like kidney epithelia, blood brain barrier, and the intestinal barrier. In the latter it is active by detoxifying bacterial derived LPS [8] and luminal bacterial derived ATP [7]. This activity of alkaline phosphatase leads to a protection of the intestinal epithelial cells against ATP and LPSinduced increases in permeability. Interestingly, alkaline phosphatase can also protect brain endothelial cells from LPS induced stress and also supports these cells in maintaining their barrier function and restores kidney function [9], likely due to barrier protection activities.

The potential detoxification of cellular stress molecules like ATP and the bacterial toxins including LPS contribute to the functional protection of the barrier functions of epithelial cell layers. This makes alkaline phosphatase an interesting candidate to test for protective effects against SIRS which potentially occurs as a side effect of cardiac cardiac bypass surgery.

The previously performed APPIRED I study was designed to investigate the safety of administrating bRESCAP to patients undergoing coronary artery bypass grafting. Patients with a low to medium mortality risk with an EuroSCORE I of 2- 6 were included in this study. The EuroSCORE 1 (European System for Cardiac Operative Risk Evaluation) has been developed to estimate perioperative mortality for patients undergoing cardiac surgery [10].

In the APPRIRED I study, patients undergoing coronary artery bypass grafting, received an intravenous bolus dose of $1000 \mathrm{IU}$ of AP just prior to surgery and in addition to that they were treated intravenously with a total dose of $200 \mathrm{IU} / \mathrm{kg}$ b.w. of AP over up to 36 hours after surgery. Potential side effects of treatment with AP and levels of inflammation were monitored in these patients. In the APPIRED I study 5 out of 31 patients in the placebo group showed a clear inflammatory response whereas in the bRESCAP group none of the patients developed an inflammatory response suggesting a protective effect of bRESCAP [11].

In the APPIRED II study patients undergoing cardiac surgery with a EuroSCORE $>5$ were randomized between bRESCAP and placebo to collect more data on the potential protective effect of bRESCAP against inflammatory reactions and on the safety of intravenous bRESCAP treatment during and after cardiac surgery.

The data of this APPIRED II study are reported here.

\section{Materials and methods}

\section{Study objectives}

The primary study endpoint was the reduction of the number of post-surgical inflammatory response in the bRESCAP treated patient group as compared to the placebo-treated group. An inflammatory response, in the sense of this study, was primarily indicated by an early increase in TNF- $\alpha$ level of at least $10 \mathrm{pg} / \mathrm{ml}$ above the pre-surgical level and a later increase in IL-6 and IL-8.

Secondary objectives of this study were 1) The reduction in organ dysfunction within 14 days of surgery 2) The duration of organ dysfunction 3) The length of organ dysfunction-associated intensive care unit (ICU) and hospital stay 4) Number of days on ventilatory support 5) Number of days of renal failure-dialysis 6) Volume of blood and blood products infused within 24 hours of surgery 7) Incidence of hospital re-admission and 8) 30-day all-cause mortality.

Safety was assessed by monitoring the incidence and severity of adverse events, vital signs and changes in clinical laboratory measurements as described under measurements.

\section{Clinical centres}

The APPIRED II study was conducted at two clinical centres: The Department of Cardiothoracic Surgery, Catharina Hospital, Eindhoven, The Netherlands and at the Anaesthesiology, Intensive Care and Emergency Medicine department, Hospital East-Limburg, Genk, Belgium.

\section{Ethics}

This study was conducted according to the principles of the Declaration of Helsinki as accepted by The World Medical Association in Seoul, October 2008 and in accordance with the Medical Research Involving Human Subjects Act (WMO).

\section{Randomization}

After signing the informed consent form, the patient was immediately admitted to the electronic case report form by entering the basic patient data (EuroSCORE 1 questionnaire). The server randomly assigned a study-specific patient ID and a treatment code to the patient.

\section{Blinding}

The responsible trial pharmacist performed randomisation of the study drugs and kept the randomisation list blinded to all other persons involved except for the clinical research organization (Aix Scientific, Aachen, Germany). During the clinical part of the study, the server computer used this list to assign the treatment codes to the patients. Both the patients and the investigators remained blinded for the treatment as received by the patients.

\section{Patients}

Patients were selected based upon the following inclusion criteria:

1. Male or non-pregnant, non-lactating female patients at the age of 18 years and older.

2. Patients scheduled for valve surgery or valve surgery in combination with CABG surgery with an EuroSCORE of $>5$. Both valve surgery patients and patients undergoing both valve surgery and CABG were included in this study as both types of surgery are prone to develop SIRS as side effect.

3. Patients who have given written informed consent prior to participation in the trial.

The exclusion criteria were:

1. Patients who were unwilling or unable to be fully evaluated for follow-up.

2. Patients who were vegetarians or veganists or those patients that were expected not to be tolerant for bovine proteins.

3. Patients who were, in the opinion of the investigator, unsuitable for the study e.g. due to comorbidities, drug use or clinical chemistry which could interfere with the interpretation of the study results. 
Keizer H (2021) Safety and efficacy data of a randomized, double-blind, placebo-controlled phase II study with the biological response modifier "bRESCAP" in patients undergoing cardiac surgery (APPIRED II)

\section{Treatment with alkaline phosphatase}

The type of alkaline phosphatase used in this study is Bovine Intestinal Alkaline Phosphatase (bIAP, bRESCAP). This enzyme is listed as EC 3.1.3.1 (Test substance name: bIAP / bovine Intestinal Alkaline Phosphatase). Its chemical name is Phosphatase, Alkaline EC 3.1.3.1.

Alkaline phosphatase was dosed as a bolus dose of 1000 IU just before anaesthesia followed by continuous infusion of $200 \mathrm{IU} / \mathrm{kg}$ b.w. of alkaline phosphatase $/ \mathrm{kg}$ dosed for 8 hours starting at surgery. In this study a unit of Alkaline phosphatase is defined as the amount of enzyme causing the hydrolysis of one micromole of p-nitrophenyl phosphate per minute at $25^{\circ} \mathrm{C}$ and $\mathrm{pH}$ 9.6.

Alkaline Phosphatase was used on top of standard care treatment.

\section{Measurements}

Efficacy and safety measurements were done before, during and after treatment. In order to evaluate the cytokine endpoints (IL-6, IL-8, IL-10, TNF- $\alpha$, MCP1, and VEGF and for C-reactive protein), blood samples were converted to serum or plasma and stored at $\leq-80^{\circ} \mathrm{C}$ for later determination.

Cytokines were measured from samples taken 1 hour before surgery, at the start of surgery and 1.5, 3,4,8,12, 24, 48.72 and 96 hours after surgery. Up to and including 24 hours after surgery, samples could be analysed in $90-100 \%$ of all patients. At 48 hrs after surgery and thereafter, the percentage of patients for which plasma samples were present decreased. At 96 hours after surgery this percentage was the lowest and was reduced to about $40 \%$ of the original numbers in both placebo and bRESCAP treated groups.

Age was reported, body mass and length were measured, and body mass index (BMI) was calculated. Blood samples were collected to evaluate various cell counts, haemoglobin, haematocrit, prothrombin time and activated partial thromboplastin time (aPTT), and to measure sodium, potassium, chloride, glucose, urea, creatinine, calcium, phosphorous, total bilirubin, total protein, albumin, alanine aminotransferase, aspartate aminotransferase, alkaline phosphatase, lactate dehydrogenase, and creatin kinase $(\mathrm{CK})$ and the CK-MB fraction.

The ICU time and the length of stay in the hospital of all patients in the study were recorded.

All adverse events occurring during the study were reported and were judged by the investigator regarding severity, and relationship to treatment.

\section{Technical procedures}

All clinical laboratory parameters, including cytokine analyses (based on the Luminex method) were determined using routine hospital protocols. All cytokine samples were measured at the same day in one batch.

\section{Statistics}

The overall difference in primary outcomes between placebo and treatment group were tested by the student T-test method. Values obtained after bRESCAP and after placebo treatment were compared. No within- group comparisons were made. In addition, a $95 \%$ confidence interval was derived.

For secondary outcomes data were analysed by appropriate parametric and non-parametric statistical procedures for a multiple sample design. All statistical tests were two-sided and were declared significant at $\mathrm{p}<0.05$.

The incidence of adverse events in placebo and treatment groups were reported. Signs and symptoms were regarded treatment related if they started on or after the date of administration of the first dose of study drug, or if they were present prior to the administration of the first dose of study drug and increased in severity during the study. Overall patient safety was evaluated by comparing the tabulated AE's in both patient groups.

\section{Results}

\section{Patient characteristics}

The inclusion and exclusion criteria as described above resulted in 62 patients signing the informed consent form and receiving a patient ID. Of these patients ultimately 27 patients were treated with bRESCAP (= bIAP = bovine intestinal alkaline phosphatase) and 26 patients with placebo. Details of these patients are presented in Table 1. Ultimately, of all patients which were initially included, 9 patients were not operated.

\section{Types of surgery}

Patients underwent CABG $(n=53)$ or CABG in combination with valve surgery $(n=52)$. The number of grafts per treatment in placebo and bRESCAP groups were similar.

\section{Patient stratification}

Mean EuroSCORE values \pm SD in the bRESCAP and placebo group was $5.81 \pm 3.09$ and $5.64 \pm 2.56$ respectively. In the bRESCAP group 5 out of 27 had a EuroSCORE $>10$ against 1 out of 26 in the Placebo group (n.s.). The left ventricular ejection fraction was $2.9+0.5$ in bRESCAP treated patients and $2.7+0.7$ in Placebo treated patients. Serum creatinine was $87.0+23.9$ in bRESCAP treated patients and 79.8 +31.9 in Placebo treated patients.

The patients in both groups were similar in baseline characteristics, although the mean BMI of the patients in the placebo group was somewhat higher in the placebo treated group than in the RESCAP treated group of patients (Table 1).

\section{Medication of patients}

As shown in Table 2, patients received various types of medication. Placebo and bRESCAP groups did not clearly differ in drug treatment.

\section{Exposure to Alkaline phosphatase}

Right after the bolus injection of 1000 IU of bRESCAP at $t=0$, alkaline phosphatase plasma concentrations raised quickly in the bRESCAP group (Figure 1). An alkaline phosphatase concentration of more than 10 times the basal value was reached. Plasma alkaline phosphatase levels decreased quickly after the bolus injection to the levels which were maintained by infusion. After 8 hours, when the infusion stopped, the alkaline phosphatase plasma levels returned to basal level within 24 hours. In the placebo group an initial decrease of

Table 1. Patient characteristics in placebo and bRESCAP groups. The BMI values of the placebo group and those of the bRESCAP group were similar in size, but statistically significantly higher in the placebo group.

\begin{tabular}{|l|c|c|c|c|c|c|}
\hline RESCAP & Mean & SD & PLACEBO & mean & SD & p-value \\
\hline Age (years) & 70.0 & 8.8 & Age (years) & 69.5 & 8.0 & 0.950 \\
\hline Height $(\mathrm{cm})$ & 170.3 & 10.4 & Height $(\mathrm{cm})$ & 168.3 & 12.4 & 0,722 \\
\hline Body Weight & 77.1 & 15.9 & Body Weight & 81.4 & 9.1 & 0,149 \\
\hline BMI & 26.5 & 4.5 & BMI & 28.9 & 3.7 & 0.007 \\
\hline
\end{tabular}


Keizer H (2021) Safety and efficacy data of a randomized, double-blind, placebo-controlled phase II study with the biological response modifier "bRESCAP" in patients undergoing cardiac surgery (APPIRED II)

Table 2. Medication provided to patients in bRESCAP and Placebo groups.

\begin{tabular}{|l|c|c|c|c|}
\hline Medication & \multicolumn{2}{|c|}{ bRESCAP } & \multicolumn{2}{c|}{ Placebo } \\
\hline Anaesthetic agents & & & & \\
\hline Fentanyl & 23 & $85.2 \%$ & 24 & $96 \%$ \\
\hline Alfentanyl & 12 & $44.4 \%$ & 14 & $56 \%$ \\
\hline Propofol & 24 & $88.9 \%$ & 25 & $100 \%$ \\
\hline Midazolam & 7 & $25.9 \%$ & 8 & $32 \%$ \\
\hline Sevoflurane & 11 & $40.7 \%$ & 10 & $40 \%$ \\
\hline Etomidate & 12 & $44.4 \%$ & 12 & $48 \%$ \\
\hline Muscle Relaxants & & & & \\
\hline Rocuronium & 15 & $55.6 \%$ & 16 & $64 \%$ \\
\hline Cisatracurium & 9 & $33.3 \%$ & 7 & $28 \%$ \\
\hline Anti-coagluating agents & & & & \\
\hline Heparin & 26 & $96.3 \%$ & 25 & $100 \%$ \\
\hline Protamine & 25 & $92.6 \%$ & 25 & $100 \%$ \\
\hline Aprotinin & 8 & $29.6 \%$ & 9 & $36 \%$ \\
\hline Anti-Arrhytmic agents & & & & \\
\hline Amiodarone or propanolol & 3 & $11.1 \%$ & 0 & $0.0 \%$ \\
\hline
\end{tabular}

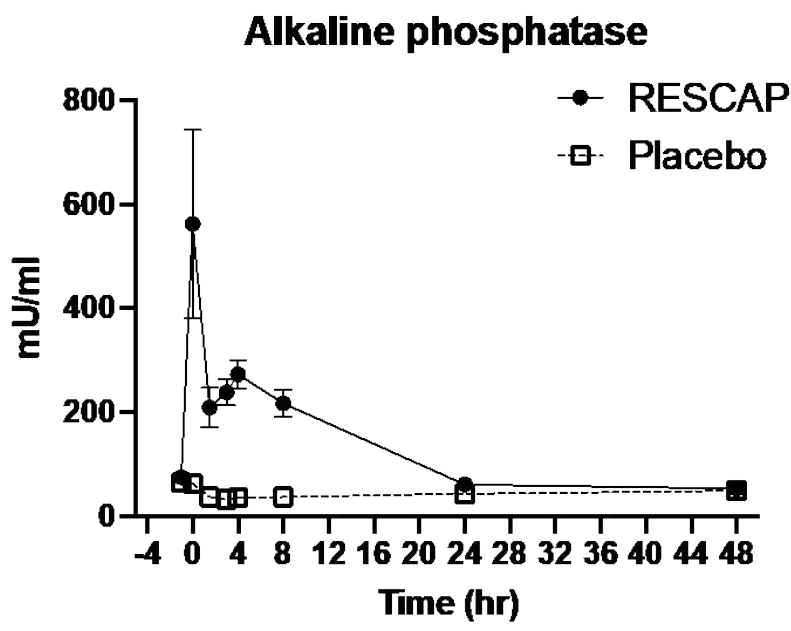

Figure 1. Plasma exposure to alkaline phosphatase in placebo group and the bRESCAP dosed group expressed as Milli-units Alkaline Phosphatase / $\mathrm{ml}$ plasma. As alkaline phosphatase is a naturally occurring enzyme, also some activity is measurable in the placebo group.

about $40 \%$ in alkaline phosphatase was observed directly after the start of surgery, which normalized later on.

\section{Effects of bRESCAP on inflammatory markers in plasma}

As shown in Figure 2, bRESCAP did not affect any of the interleukin responses as induced by surgery when compared to placebo responses.

For TNF-alpha, a positive response was defined per protocol as an increase of $10 \mathrm{pg}$ TNFalpha / $\mathrm{ml}$ plasma over pre-operation values. In none of the patients this plasma level of TNF-alpha was reached. In this study TNF-alpha plasma levels did not respond to surgery. The other cytokines showed a temporarily response to surgery.

For IL8 there appears to be a difference in plasma levels between placebo and bRESCAP treated groups starting at 48 hours after surgery (n.s.). However, it is important to note that these differences started to occur at 48 hours after surgery and later. At these time points the exposure to alkaline phosphatase was no longer different between the groups. Furthermore, these differences were the result of 1 patient having a high isolated IL8 value at 48 hours after surgery and another patient having a high IL8 values at 72 and 96 hours after surgery. These two patients were not remarkable in any other aspect in this study. In other words, the apparent effects of alkaline phosphatase on plasma IL 8 levels are probably chance findings.

Four patients had increased pre-operation values for IL6, IL8, IL10 and/or TNF alpha. The plasma levels of IL6, IL8, IL10, and TNF alpha of these patients were not used to prepare the graphs of Figure 2 as the data as obtained from these patients obscured the effect of surgery on these parameters.

Summarizing, plasma levels of TNF alpha, IL10, IL8 IL6, VEGF and MCP1 did not show a statistically significant change upon treatment with bRESCAP compared to placebo, although various of these inflammatory markers did temporarily increase upon cardiac surgery.

\section{Clinical observations}

Surgery: Surgery lasted $4.7 \pm 1.4$ hours in both treatment groups (n.s.). The perfusion time was $134 \pm 40$ minutes in the placebo group and $135 \pm 41$ minutes in the bRESCAP group (n.s.). The cross-clamping time was $109 \pm 44$ minutes in the placebo group and $100 \pm 36$ minutes in the bRESCAP group (n.s.)

Duration of stay in intensive care unit / hospital: The mean duration of stay at the intensive care unit was 4.7 days and the mean duration of stay in the hospital was 12 days in both groups (n.s.).

Mortality: In the bRESCAP treated group of 27 patients, no mortality occurred ( $0 \%)$. In the placebo group of 26 patients, 3 patients died (11.5\%) (n.s.)

The first of these patients had a EuroSCORE of 8. His interleukin levels were nonremarkable with the exception of IL6 which was slightly increased at 12 hours after surgery. This IL6 level had normalized at 24 hours after surgery. At day 4 after surgery kidney failure started, ultimately culminating in multiorgan failure which caused his death at day 17 after surgery. His multi-organ failure was probably caused by a poor left and right vertricular function resulting in cardiogenic shock.

The second patient who died had a EuroSCORE of 6.0. She was one of the few patients which showed an effect on TNF alpha. TNF alpha plasma levels peaked at 4 hours after surgery (at $4.6 \mathrm{pg} / \mathrm{ml}$ ), and normalized within 8 hours after surgery. At 4 hours after surgery also IL6, MCP-1 and IL8 levels were increased in this patient to a level 2 to 5 to times higher than observed in the other patients. At 8 hours after surgery, these values had normalized to non-remarkable values. One day after surgery, when her cytokines had normalized, she got a large myocardial infaction resulting in a decreased left ventricular ejection fraction, resulting in cardiogenic shock. At day 14 after surgery she died of ventricular fibrillation.

The last patient who died was had an EuroSCORE of 12.0. Up to and including day 2 after surgery her cytokine levels were nonremarkable. Thereafter, pulmonary distress developed which proved to be caused by pneumonia. Starting at 3 days after surgery her cytokine levels strongly increased (with the exception of TNFalpha and VEGF) to levels more than 10 times the values as observed in the other patients at day 5 after surgery. At day 6 after surgery she died of septic shock and multiorgan failure.

Cardiac function: During the post operation observation period, the mean heart rate, body temperatures and (systolic and diastolic) blood pressure were not statistically different in both groups (Figure 3).

Adverse events after surgery: In total 8 patients of the placebo treated group of patients suffered from various serious adverse effects. Three of those died under conditions as described under "Mortality". In total 5 patients who survived in both groups suffered from various serious adverse events related to either surgery or their clinical 
TNF-alpha

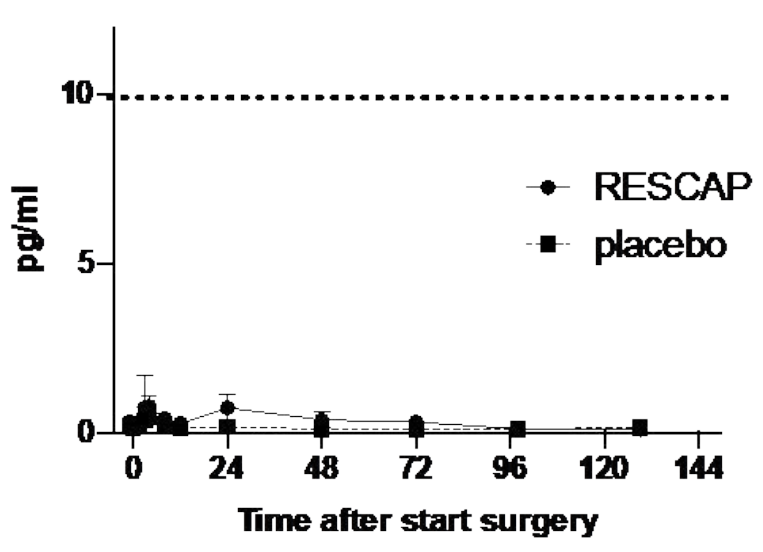

II-8

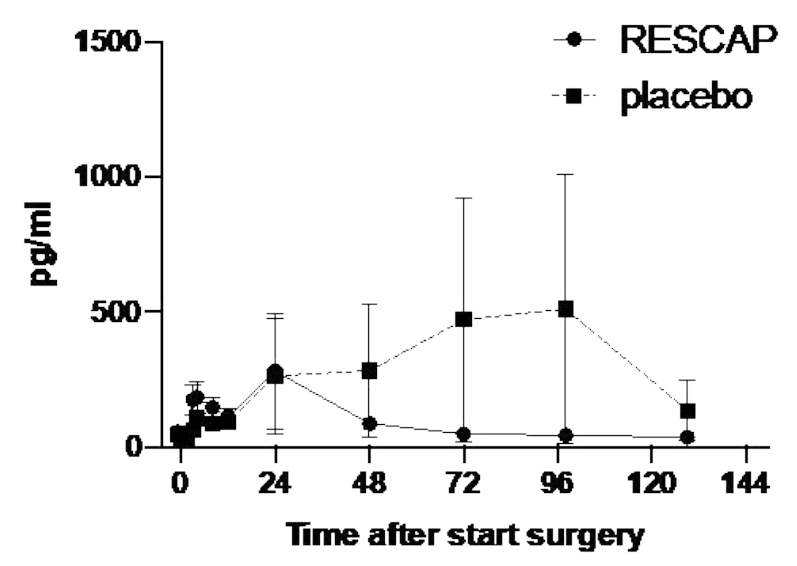

MCP

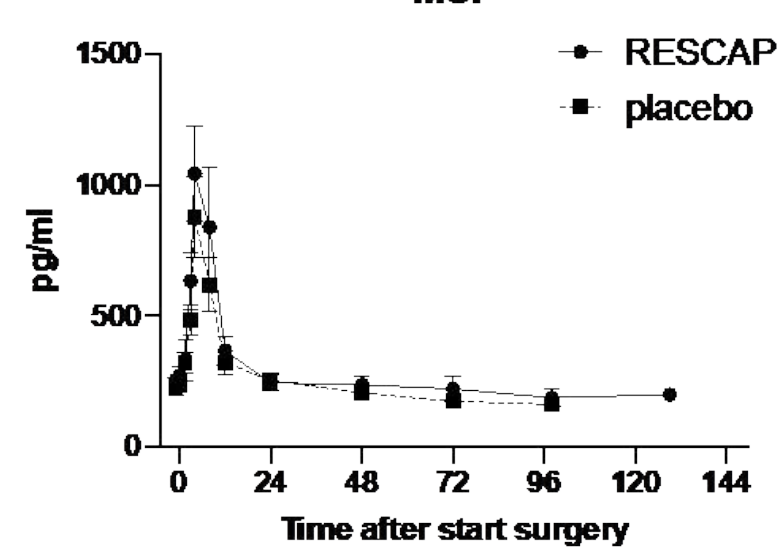

||-10

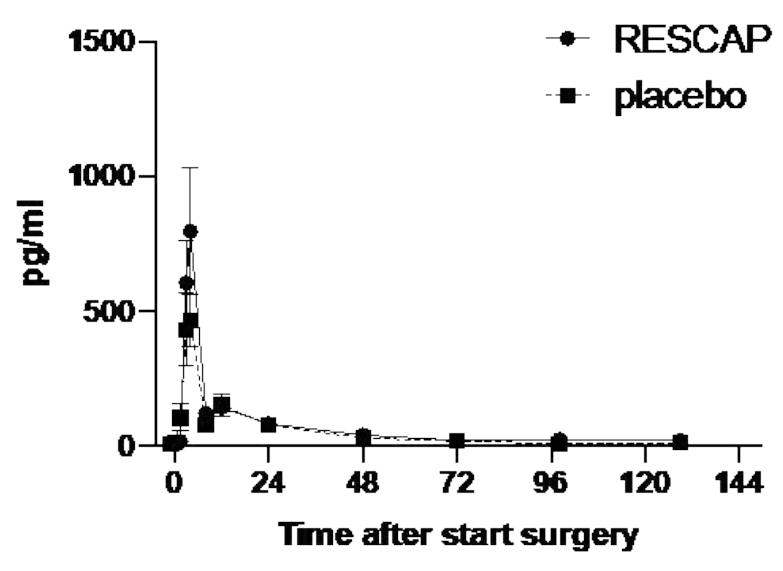

IL-6

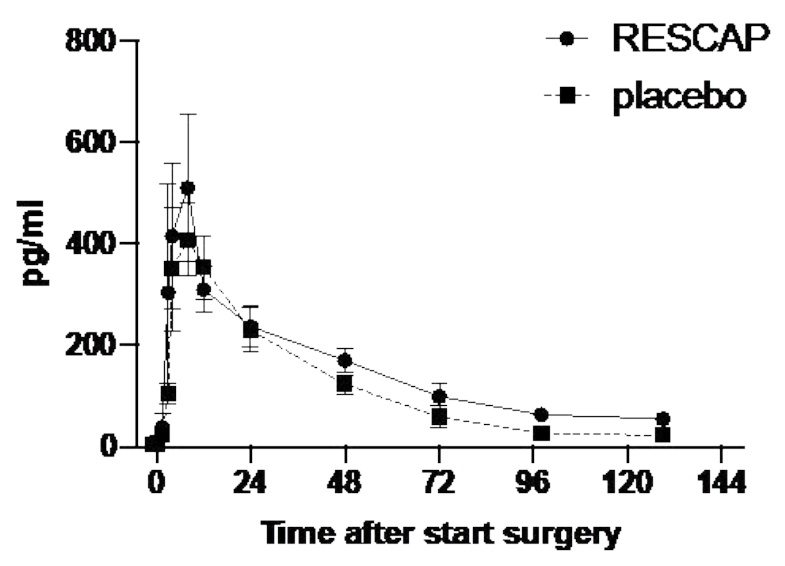

VEGF

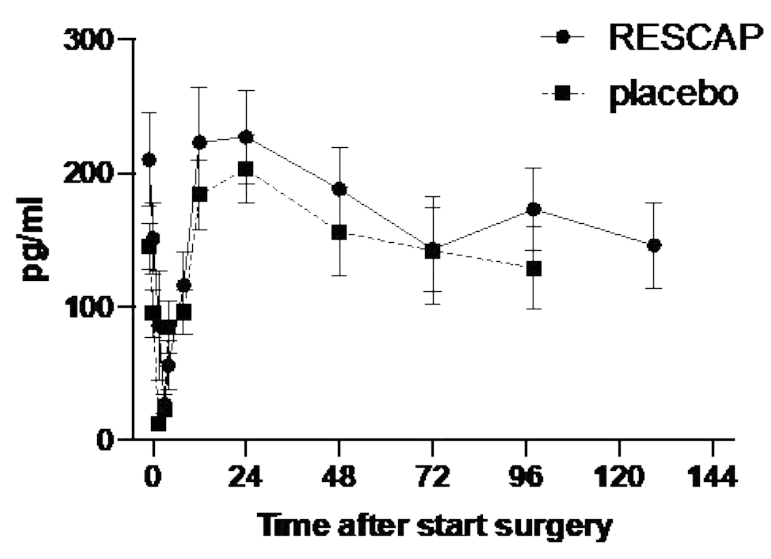

Figure 2. Plasma levels of inflammatory markers before and right after surgery. For TNF-alpha, a threshold value of $10 \mathrm{pg} / \mathrm{ml}$ was defined per protocol as a positive response. This value has been marked with a dotted horizontal line. 

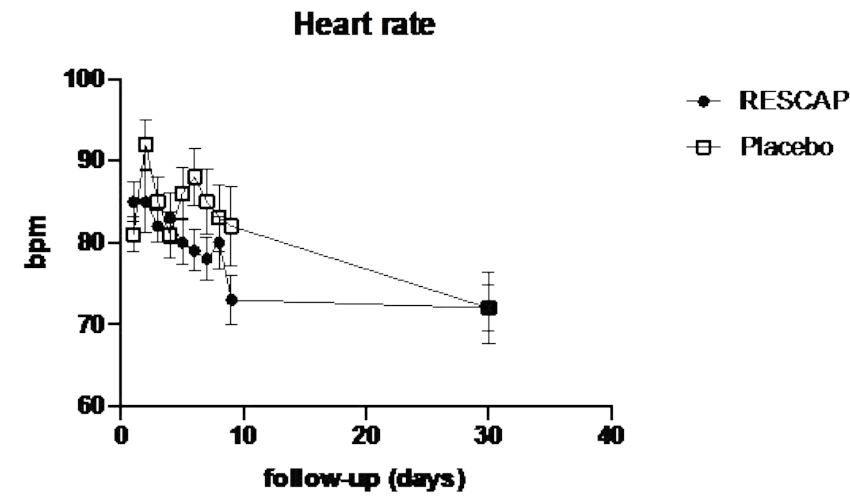

Systolic blood pressure
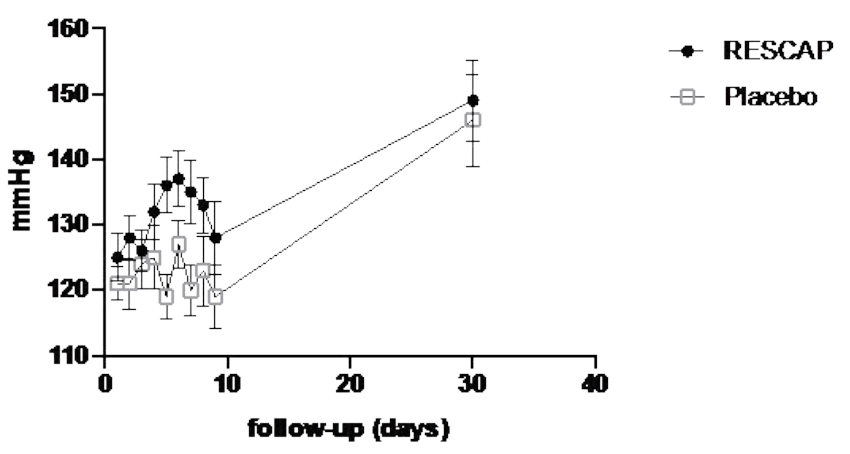

body temperature

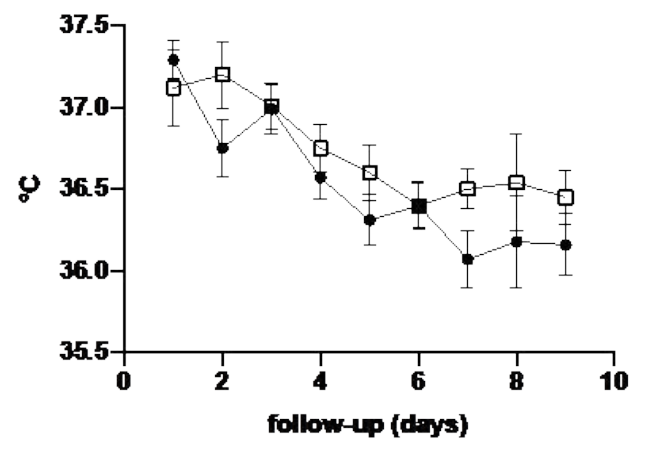

- RESCAP

$\square$ Placebo

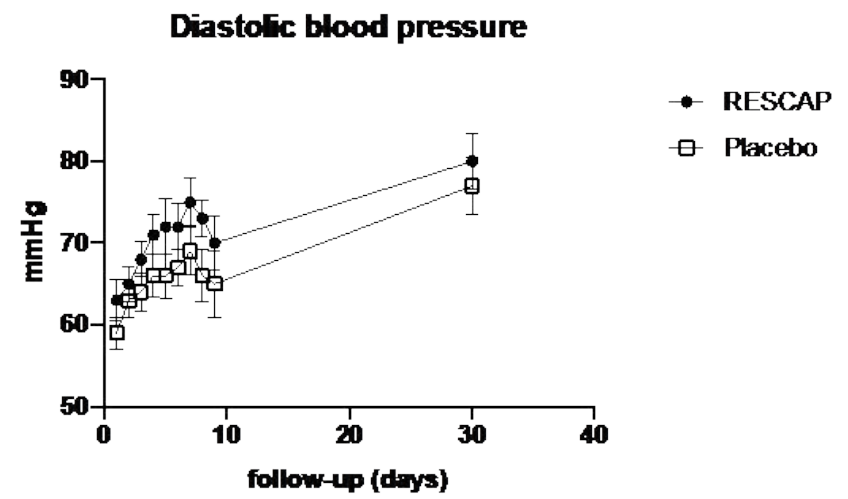

Figure 3. Heart rate, body temperature and (systolic and diastolic) blood pressure upon follow up of the study.

condition. The total number of serious events in both groups were not statistically significantly different from each other.

Clinical chemistry parameters: No statistically significant differences were observed between the bRESCAP treated and placebo treated groups regarding clinical chemistry parameters.

\section{Discussion}

The APPRIED II study was initiated to confirm and extent safety and efficacy data for bRESCAP as obtained in a previously conducted clinical trial (APPIRED I) in patients undergoing coronary artery bypass grafting with the use of cardiopulmonary bypass [11].

The APPIRED II study was performed in patients undergoing both coronary artery grafting and valve replacement. This more timeconsuming surgery increased the cross-clamping time from a mean value of 47 minutes in APPIRED I to a mean value of 109 minutes in APPIRED II. As the cross-clamping time appears to be related to circulating LPS concentrations during cardiac surgery [12] and cross clamping times over 98 minutes seem related to gastrointestinal complaints during cardiac surgery [13] it was suggested that the chance of SIRS-like inflammatory reactions would be increased in APPIRED II.

In reality however, plasma levels of TNF-alpha were not increased in this study in both the bRESCAP and the placebo groups. As in this study plasma levels of TNF alpha did not increase upon surgery and basal TNF alpha levels were at detection level, it was not possible to show a decrease of TNFalpha as we had expected to see after treatment with bRESCAP.
Although no effects were found on TNF alpha plasma levels, inflammatory responses to surgery were observed for most of the other cytokines. These responses were probably caused by direct activation of white blood cells [3] and/or by general endothelial stress due to surgical procedure and the use of cardiopulmonary bypass [2]. None of these factors are likely to be influenced by bRESCAP treatment.

For IL8, at 48 hours after surgery and later, plasma values seemed higher in patients treated with placebo. However, this apparent effect was caused by high IL8 values in single patients at various time points and these differences occurred at 24 hours after the moment that exposure to alkaline phosphatase had dropped to control values in the bRESCAP group. Therefore, it seems unlikely that the observed difference in IL8 plasma levels between the two groups was caused by a protective effect of bRESCAP.

During the whole post-surgery observation period, the mean hart rate was consistently lower, and the mean systolic and diastolic blood pressure were consistently higher in patients treated with bIAP than in patients of the placebo group. It is possible that these apparent haemodynamic effects may reach statistical significance in a study with a larger sample size. If so, this may indicate a better recovery of cardiac function in bIAP treated patients.

In APPIRED II, 3 patients died. All 3 deaths were observed in the placebo group and none in the bRESCAP group. We consider it unlikely that any of these deaths could have been prevented if these patients would have been treated with bRESCAP instead of placebo, as the lethal events occurred later than the 8 hours after surgery during which bRESCAP had been dosed. Furthermore, lethality did not seem to be related to a 
Keizer H (2021) Safety and efficacy data of a randomized, double-blind, placebo-controlled phase II study with the biological response modifier "bRESCAP" in patients undergoing cardiac surgery (APPIRED II)

surgery induced effects on inflammatory factors which may have been prevented by bRESCAP treatment [11].

In less than $1 \%$ of the patients undergoing coronary artery bypass grafting, intestinal ischemia develops during surgery $[12,13]$. This serious condition with high mortality, is expected to be well responsive to bRESCAP as intestinal ischemia results in systemic exposure to LPS [14] and systemic exposure ATP due to large scale tissue damage probably play an important role in this condition [15], and bRESCAP detoxifies both.

Nilsson et al. [5] evaluated the EuroSCORE levels of cardiac surgery patients who did or did not develop intestinal ischemia and found that in their patients without intestinal ischemia had a mean EuroSCORE of around 5, whereas the patients who did develop intestinal ischemia had a mean EuroSCORE of 8 . As the APPIRED II study had a mean EuroSCORE of 5.3, and a group size of less than 30, it is perhaps not surprising that no intestinal ischemia and associated cytokine responses developed shortly after surgery in this study.

In order to get a proper view of the potential efficacy of bRESCAP in preventing SIRS and or life-threatening intestinal ischemia in cardiac surgery, the APPIRED III study has started with a much larger number of patients having higher EuroSCORE values.

Safety wise, APPIRED II was successful. Intravenous treatment with bRESCAP, leading to clearly increased plasma levels of alkaline phosphatase, did not result in any adverse effect regarding inflammatory parameters, clinical chemistry parameters, clinical haematology or on any of the other clinical observations performed.

As total plasma alkaline phosphatase levels were more than 4 times increased over basal levels during intravenous bRESCAP infusion, but no adverse effects of any kind were noted in this study it can be concluded that intravenous treatment with bRESCAP of human cardiac surgery patients at this dose level is safe.

\section{Conclusion}

The APPIRED II study was conducted to show an anti-inflammatory effect of intravenous bRESCAP treatment and to confirm the safety of intravenous bRESCAP in cardiac surgery patients with a EuroSCORE $>5$.

The APPIRED II study was inconclusive regarding the prevention of an TNF alpha dependent inflammatory reaction in cardiac surgery patients as no such response occurred in placebo treated cardiac surgery patients.

The APPIRED II study was successful in showing that intravenous treatment with alkaline phosphatase leading to clearly elevated plasma levels was safe.

\section{CrediT author statement}

Hiskias Keizer: Writing- original draft preparation and data curation. Suzanne Kats: Investigation, Ines Christis: Investigation,
Rene Heylen: Investigation, Ronald Oosting: Writing - review and editing, Willem Seinen: Writing - review and editing and Ruud Brands: Supervision, funding acquisition, conceptualization.

\section{Funding}

This study was sponsored by Alloksys, Agro Business Park 10, 6708PW Wageningen, The Netherlands.

\section{References}

1. Cremer J, Martin M, Redl H, Bahrami S, Abraham C, et al. (1996) Systemic inflammatory response syndrome after cardiac operations. Ann Thorac Surg 61: 17141720. [Crossref]

2. Larmann J, Theilmeier G (2004) Inflammatory response to cardiac surgery: cardiopulmonary bypass versus non-cardiopulmonary bypass surgery. Best Pract Res Clin Anaesthesiol 18: 425-438. [Crossref]

3. Habbal EL, Carter H, Smith LJ, Elliott MJ, Strobel S (1995) Neutrophil activation in paediatric extracorporeal circuits: effect of circulation and temperature variation. Cardiovascular research 29: 102-107. [Crossref]

4. Oudemans-van Straaten HM, Jansen PG, Hoek FJ, van Deventer SJ, Sturk A, et al. (1996) Intestinal permeability, circulating endotoxin, and postoperative systemic responses in cardiac surgery patients. J Cardiothorac Vasc Anesth 10: 187-194. [Crossref]

5. Nilsson L, Kulander L, Nyström SO, Eriksson O (1990) Endotoxins in cardiopulmonary bypass. J Thorac Cardiovasc Surg 100: 777-780. [Crossref]

6. Poelstra K, Bakker WW, Klok PA, Hardonk MJ, Meijer DK (1997) A physiologic function for alkaline phosphatase: endotoxin detoxification. Lab Invest 76: 319. [Crossref]

7. Malo MS, Moaven O, Muhammad N, Biswas B, Alam SN, et al. (2014) Intestinal alkaline phosphatase promotes gut bacterial growth by reducing the concentration of luminal nucleotide triphosphates. Am J Physiol Gastrointest Liver Physiol 306: G826-G838. [Crossref]

8. Liu W, Hu D, Huo H, Zhang W, Adiliaghdam F, et al. (2016) Intestinal alkaline phosphatase regulates tight junction protein levels. J Am Coll Surg 222: 1009-1017. [Crossref]

9. Heemskerk S, Masereeuw R, Moesker O, Bouw M, van der Hoeven JG, et al. (2007) Alkaline phosphatase treatment improves renal function in patients with severe sepsis or septic shock. Critical Care 11: P14. [Crossref]

10. Nashef SA, Roques F, Michel P, Gauducheau E, Lemeshow S, et al. (1999) European system for cardiac operative risk evaluation (Euro SCORE). Eur J Cardiothorac Surg 16: 9-13. [Crossref]

11. Suzanne K (2009) Anti-inflammatory effects of alkaline phosphatase in coronary artery bypass surgery with cardiopulmonary bypass. Recent Pat Inflamm Allergy Drug Discov 3: 214-220. [Crossref]

12. Nilsson J, Hansson E, Bodil A (2013) Intestinal ischemia after cardiac surgery: analysis of a large registry. J Cardiothorac Surg 8: 156. [Crossref]

13. Lütfi S, Aydın OU, Yıldız M, Serdaroğlu H, Kurtoğluet M, et al. (2019) Comparison of intestinal ischemia after on-pump versus off-pump coronary artery bypass grafting surgery. Turk J Med Sci 49: 11-15. [Crossref]

14. Gathiram P, Wells MT, Raidoo D, Brock-Utne JG, Gaffin SL (1989) Changes in lipopolysaccharide concentrations in hepatic portal and systemic arterial plasma during intestinal ischemia in monkeys. Circ shock 27: 103-109. [Crossref]

15. Cauwels A, Rogge E, Vandendriessche B, Shiva S, Brouckaert P (2014) Extracellular ATP drives systemic inflammation, tissue damage and mortality. Cell Death Dis 5: e1102-e1102. [Crossref]

Copyright: (C2021 Keizer H. This is an open-access article distributed under the terms of the Creative Commons Attribution License, which permits unrestricted use, distribution, and reproduction in any medium, provided the original author and source are credited. 